\title{
Two-scales kinetic theory model of short-fibers aggregates
}

\author{
Emmanuelle Abisset-Chavanne ${ }^{1, \mathrm{a}}$, Rabih Mehzer $^{1, \mathrm{~b}}$, and Fransisco Chinesta ${ }^{1, \mathrm{c}}$ \\ GeM, UMR CNRS-Centrale Nantes, \\ 1 , rue de la Noë, BP92101, \\ F-44321 Nantes Cedex 3, France \\ aemmanuelle.abisset-chavanne@ec-nantes.fr, ${ }^{\mathrm{a}}$ rabih.mezher@ec-nantes.fr, \\ cfransisco.chinesta@ec-nantes.fr
}

Keywords: CNTs suspension, kinetic theory, multiscale modelling, numerical simulation

\begin{abstract}
This paper proposes a first attempt to define a two scales kinetic theory to describe concentrated suspensions involving short fibers, nano-fibers or nanotubes. In this case, fiber-fiber interactions can not be neglected and rich microstructures issued from these interactions can be observed, involving a diversity of fibers clusters or aggregates with complex kinematics, and different sizes and shapes. These clusters can interact to create larger clusters and also break because the flow induced hydrodynamic forces. In this paper we propose a double-scale model to describe such microstructure: at the finest scale we study the cluster kinematic based on the behaviour of the rods that constitute it, at a coarser scale, we use clusters distribution to derive the effect of the clusters presence on the suspensions properties.
\end{abstract}

\section{Introduction}

In order to improve some fluid mechanical or thermal properties, one solution is to introduce nanoparticules that change the fluid behaviour and properties, we talk then about "charged fluid". A challenge is then to be able to predict the new properties of the fluid depending on the type of particles and flow induced microstructure evolution on which depends the macroscopic behaviour. In this work, we focus on suspensions involving rods more or less concentrated.

Depending on this concentration, the rods -- short fibers, nanofibers or CNTs -- suspensions present different morphologies. When the concentration is dilute enough the microstructure can be described by tracking a population of rods that move with the suspending fluid and orient depending on the velocity gradient according to the Jeffery's equation [1] that relates the orientation evolution with the flow velocity field. In that case the motion and orientation of each fiber is assumed decoupled from the others. As soon as the concentration increases the difficulties appear. In the semi-dilute or semiconcentrated regimes fiber-fiber interactions occur, but in general they can be accurately modelled by introducing a sort of randomizing diffusion term. The worst scenario that we are going to focus on in this paper concerns concentrated suspensions involving clusters of entangled particles and in which aggregation/disaggregation take place.

At the macroscopic scale, a possible way to describe such a charged fluid is to try to fit some powerlaw constitutive equation. However, this description does not allow to describe the microstructure. At the microscopic scale, direct numerical simulations describing complex fiber-fiber interactions can be carried out in small enough representative volumes. However, such a scale does not allow real scale problems resolution. Finally, a natural candidate to be a reasonable compromise between (fine) micro and (fast) macro descriptions consists of considering a mesoscale model, developed in the framework of kinetic theory.

The main advantage of such kinetic theory approaches [2] [3] is their capability to address macroscopic systems, while keeping the fine physics through a number of conformational coordinates introduced for describing the microstructure and its time evolution. At this mesoscopic scale, the microstructure is defined from a distribution function that depends on the physical space, the time and a number of conformational coordinates. The moments of this distribution constitutes a coarser description, in general used in macroscopic modelling [4]. 
Thanks to the use of this approach, the charged fluid model will include two scales: the one involving the aggregates and the one related to the rods constituting the aggregates.

In this paper, we first develop the description of suspensions involving rigid clusters, before moving to the description on suspensions involving deformable clusters. Indeed, the study of the rigid clusters allows to define a rigidity term in the deformable cluster modelling. Thus, for both case, starting from a microscopic description of the clusters equilibrium, we move to the mesoscopic one using the kinetic theory approach. Finally, the fluid virial stresses due to the suspensions is derived and, in a last section, compared to experimental results on the apparent viscosity of CNTs suspensions.

\section{Suspension involving rigid clusters}

When the concentration of rods in a suspension is high enough, a sort of clusters composed of entangled rods is observed. When this suspension flows, those clusters seem animated of almost rigid motions. The objective of this section is to derive a first model, starting from the microscale, allowing to describe the clusters' evolution and their influence on the virial stress of the fluid.

Clusters can be sketched in two dimensions as entangled aggregates of rods (see Fig. 1). These rods can be idealized by rigid segments joining two opposite beads.

In order to define a simple -- but realistic -- geometry for the clusters, we extract the beads located on the periphery of the cluster as illustrated in Fig. 2. Then we consider the star configuration depicted in Fig. 3 that will be the cluster idealization on which we are performing the kinematic and dynamic analysis.

Obviously, this process constitutes a tremendous simplification but it constitutes the simplest model that could be enriched without major difficulties to cover more complex and rich systems.

\section{Microscopic description of a rigid cluster composed of rods}

We consider a rigid cluster consisting of $N$ rods, oriented in the directions $\mathbf{p}_{i}$ as sketched in Fig. 4. In this study, Brownian effects can be neglected and then only flow induced hydrodynamic forces are considered. The rods are assumed to be inertialess and we also assume that the force only applies on the beads. In order to derive the kinematic of the rigid cluster, we first write its equilibrium.

For a cluster subjected to only hydrodynamic forces, a force $\mathbf{F}_{i}$ applies on each bead $L \cdot \mathbf{p}_{i}$, assumed given by:

$$
\mathbf{F}_{i}=\xi \cdot\left(\nabla \mathbf{v} \cdot \mathbf{p}_{i} \cdot L-\dot{\mathbf{p}}_{i} \cdot L\right)
$$

It can be noticed that this expression consists of two contributions: a sort of affine deformation given by the term $\nabla \mathbf{v} \cdot \mathbf{p}_{i}$ and the second one that removes the deformation in the rod direction because, in presence of other force, the rod is considered inextensible. The coefficient $\xi$ can be seen as a viscosity of the fluid: if $\xi$ is low, the fluid does not have much influence on the cluster kinematics, and on the opposite for large $\xi$, the orientation of the cluster will be strongly influenced by the fluid flow. The value $\xi=0$ is a non-physical one and should be never considered.

Now, as the rods and so the cluster are inertialess, we enforce a null resulting moment for the whole cluster.

The moment associated with rod $i$ is given by

$$
\mathbf{M}_{i}=2 \cdot L \cdot \mathbf{p}_{i} \times \mathbf{F}_{i}
$$

where the symbol " $\times$ " denotes the cross product.

The forces are by construction self-equilibrated for the whole cluster, thus we only need to enforce the nullity of the resulting moment: 


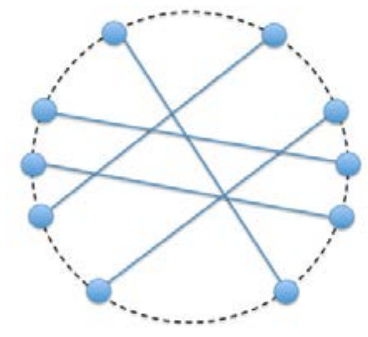

Fig. 1: Entangled rods constituting a rigid cluster

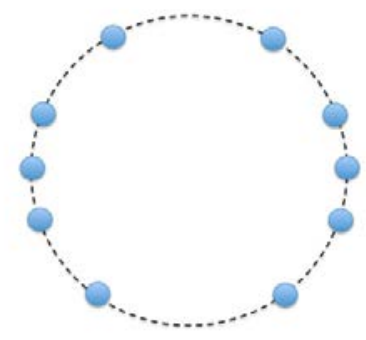

Fig. 2: Extracting the active beads located on the cluster periphery

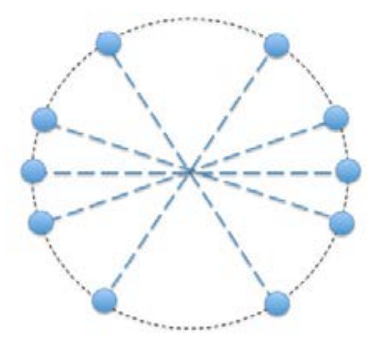

Fig. 3: Defining the rigid star representation

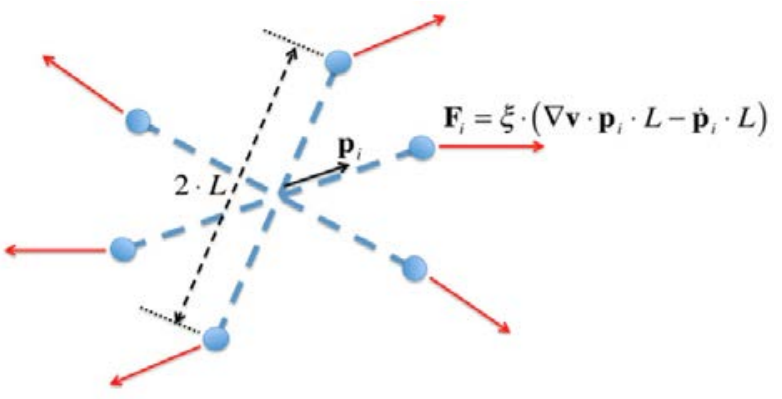

Fig. 4: Hydrodynamic forces applying on a rigid cluster composed of rods 


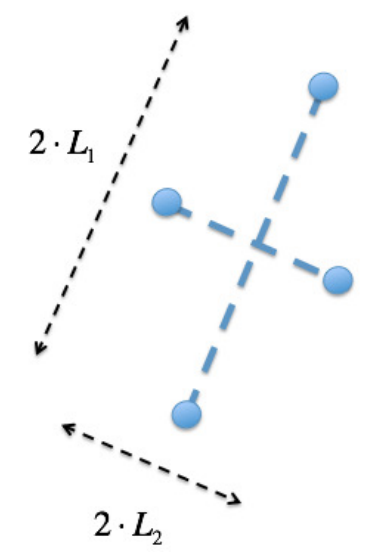

Fig. 5: Two-rods rigid cluster representing an ellipsoidal 2D particle

$$
\sum_{i=1}^{i=N} \mathbf{M}_{i}=\mathbf{0}
$$

If we define the cluster angular velocity $\boldsymbol{\omega}$ such that

$$
\dot{\mathbf{p}}_{i}=\boldsymbol{\omega} \times \mathbf{p}_{i}
$$

then, in the 2D case, and because $\boldsymbol{\omega}$ and $\mathbf{p}_{i}$ are orthogonal, $\mathbf{p}_{i} \times \dot{\mathbf{p}}_{i}=\boldsymbol{\omega} \forall i$, the kinematics of the rigid $2 \mathrm{D}$ cluster can be defined from:

$$
\boldsymbol{\omega}=\frac{\sum_{i=1}^{i=N} \mathbf{p}_{i} \times\left(\nabla \mathbf{v} \cdot \mathbf{p}_{i}\right)}{N}
$$

being the angular velocity of each $\operatorname{rod} j$ :

$$
\dot{\mathbf{p}}_{j}=\boldsymbol{\omega} \times \mathbf{p}_{j}=\frac{\left(\sum_{i=1}^{i=N} \mathbf{p}_{i} \times\left(\nabla \mathbf{v} \cdot \mathbf{p}_{i}\right) \times \mathbf{p}_{j}\right.}{N}
$$

By simple manipulations on these relations, we can prove that in order to represents non-infinite aspect ratio 2D particles, it suffices to consider a rigid system composed of two rods, aligning perpendicularly the one with respect to the other, and having lengths $2 \cdot L_{1}$ and $2 \cdot L_{2}, L_{1} \geq L_{2}$, as depicted in Fig. 5. Moreover, in [5], the author proves that this description allows to find the Jeffery's expression for an ellipsoid of aspect ratio $\frac{L_{1}}{L_{2}}$.

\section{Mesoscopic description of a rigid cluster composed of rods}

In the microscopic description, the cluster is described by $\mathrm{N}$ discrete rods studied individually. To define the mesoscopic description, an alternative description consists in defining its orientation distribution $\psi(\mathbf{p})$. Then, all the discrete sums on $\mathrm{N}$ of the microscopic description can be substituted by the corresponding integrals weighted with the distribution function $\psi$.

Thus in the 2D case Eq. (5) leads to:

$$
\boldsymbol{\omega}=\int_{\mathcal{S}(0,1)} \mathbf{p} \times(\nabla \mathbf{v} \cdot \mathbf{p}) \cdot \psi(\mathbf{p}) d \mathbf{p}
$$

being the angular velocity of any rod $\mathbf{q}$ : 


$$
\dot{\mathbf{q}}=\boldsymbol{\omega} \times \mathbf{q}=\int_{\mathcal{S}(0,1)}(\mathbf{p} \times(\nabla \mathbf{v} \cdot \mathbf{p})) \times \mathbf{q} \cdot \psi(\mathbf{p}) d \mathbf{p}
$$

Because $(\mathbf{p} \times(\nabla \mathbf{v} \cdot \mathbf{p}))_{i}=\boldsymbol{\epsilon}_{i j k} \cdot \mathbf{p}_{j} \cdot(\nabla \mathbf{v})_{k l} \cdot \mathbf{p}_{l}$, it is easy to prove that Eq. (7) writes:

$$
\boldsymbol{\omega}=\boldsymbol{\epsilon}:\left(\mathbf{a} \cdot(\nabla \mathbf{v})^{T}\right)
$$

with a the second moment of the orientation distribution function.

On the other hand we can define the rotation tensor $\mathbf{W}$ such that

$$
\omega \times \mathbf{p}=\mathbf{W} \cdot \mathbf{p}
$$

that allows writting

$$
\dot{\mathbf{p}}=\mathbf{W} \cdot \mathbf{p}
$$

Thus, the mesoscopic description uses:

$$
\left\{\begin{array}{l}
\boldsymbol{\omega}=\boldsymbol{\epsilon}:\left(\mathbf{a} \cdot(\nabla \mathbf{v})^{T}\right) \\
\dot{\mathbf{p}}=\boldsymbol{\omega} \times \mathbf{p} \equiv \mathbf{W} \cdot \mathbf{p} \\
\mathbf{W}=\mathbf{W}(\boldsymbol{\omega})
\end{array}\right.
$$

These equations show that a rigid cluster rotates with a velocity that only depends on the second moment of its orientation distribution function a. Then, any cluster having the same a will have the same rotational velocity. The cluster kinematics can then be described by the evolution of a, taking its time derivative.

Using the definition of a and the Eq. (12), it results:

$$
\begin{aligned}
& \dot{\mathbf{a}}=\int_{\mathcal{S}(0,1)}((\mathbf{W} \cdot \mathbf{p}) \otimes \mathbf{p}+\mathbf{p} \otimes(\mathbf{W} \cdot \mathbf{p})) \cdot \psi(\mathbf{p}) d \mathbf{p}= \\
& =\mathbf{W} \cdot \mathbf{a}+\mathbf{a} \cdot \mathbf{W}^{T}
\end{aligned}
$$

Remark: the objectivity of $\dot{a}$ has been proven in [5], along with the non necessity of a closure relation.

\section{Global description of a suspension involving several rigid clusters}

The mesoscopic description has shown that rigid clusters rotate with a velocity that only depends on the second moment a of its orientation distribution function $\psi(\mathbf{p})$. This fact suggests that a microstructure involving suspensions of several rigid clusters can be based on a distribution function $\Psi(\mathbf{x}, t, \mathbf{a})$ that represents the fraction of clusters that at position $\mathbf{x}$ and time $t$ have a configuration described by a related to the orientation of the rods composing it and that was noted by $\psi(\mathbf{p})$.

\section{Virial stress induced by clusters suspension}

The objective of this part is to determine the virial stress in the suspension due to the presence of clusters.

Contribution of a unique rigid cluster At the microscopic scale, the contribution of a rigid cluster composed of $N$ rods to the virial stress results:

$$
\boldsymbol{\tau}=\xi \cdot L^{2} \cdot \sum_{i=1}^{N} \mathbf{p}_{i} \otimes\left(\nabla \mathbf{v} \cdot \mathbf{p}_{i}-\dot{\mathbf{p}}_{i}\right)
$$


that taking into account the expression of $\dot{\mathbf{p}}_{i}$ reads:

$$
\boldsymbol{\tau}=\xi \cdot L^{2} \cdot \sum_{i=1}^{N} \mathbf{p}_{i} \otimes\left(\nabla \mathbf{v} \cdot \mathbf{p}_{i}-\mathbf{W} \cdot \mathbf{p}_{i}\right)
$$

or

$$
\boldsymbol{\tau}=\xi \cdot L^{2} \cdot \sum_{i=1}^{N}\left(\left(\mathbf{p}_{i} \otimes \mathbf{p}_{i}\right) \cdot(\nabla \mathbf{v})^{T}-\left(\mathbf{p}_{i} \otimes \mathbf{p}_{i}\right) \cdot \mathbf{W}^{T}\right)
$$

In the continuous mesoscopic framework Eq. (16) results:

$$
\begin{gathered}
\boldsymbol{\tau}^{i}=2 \cdot N_{p} \int_{\mathcal{S}(0,1)}\left((\mathbf{p} \otimes \mathbf{p}) \cdot(\nabla \mathbf{v})^{T}-(\mathbf{p} \otimes \mathbf{p}) \cdot \mathbf{W}^{T}\right) \cdot \psi(\mathbf{p}) d \mathbf{p}= \\
=2 \cdot N_{p} \cdot\left(\mathbf{a} \cdot(\nabla \mathbf{v})^{T}-\mathbf{a} \cdot \mathbf{W}^{T}\right)=2 \cdot N_{p} \cdot\left(\mathbf{a} \cdot(\mathbf{D}+\boldsymbol{\Omega})^{T}-\mathbf{a} \cdot(\boldsymbol{\Omega}+\mathbf{F})^{T}\right)= \\
=2 \cdot N_{p} \cdot(\mathbf{a} \cdot \mathbf{D}+\mathbf{a} \cdot \mathbf{F})
\end{gathered}
$$

where

$$
\left\{\begin{array}{l}
\mathbf{W}=\Omega+F \\
\mathbf{F}=\left(\begin{array}{cc}
0 & -f \\
f & 0
\end{array}\right) \\
f(\mathbf{D}, \mathbf{a})=-\mathbf{D}_{12}-2 \cdot \mathbf{D}_{11} \cdot \mathbf{a}_{12}+2 \cdot \mathbf{D}_{12} \cdot \mathbf{a}_{11}
\end{array}\right.
$$

The identity $\mathbf{D} \cdot \mathbf{a}-\mathbf{F} \cdot \mathbf{a}=\mathbf{a} \cdot \mathbf{D}+\mathbf{a} \cdot \mathbf{F}$ ensures the stress tensor symmetry, i.e. $\boldsymbol{\tau}=\boldsymbol{\tau}^{T}$.

Contribution of all the rigid clusters Now, by considering all the clusters that contribute to the local stress,

$$
\boldsymbol{\tau}=2 \cdot \tilde{N}_{p} \cdot \int_{\mathcal{A}}(\mathbf{D} \cdot \mathbf{a}-\mathbf{F} \cdot \mathbf{a}) \cdot \Psi(\mathbf{a}) d \mathbf{a}
$$

In order to avoid spurious non-symmetries when solving the kinetic model by using stochastic simulations we could consider a symmetrized form of the virial stress:

$$
\boldsymbol{\tau}=\tilde{N}_{p} \cdot \int_{\mathcal{A}}(\mathbf{D} \cdot \mathbf{a}+\mathbf{a} \cdot \mathbf{D}-\mathbf{F} \cdot \mathbf{a}+\mathbf{a} \cdot \mathbf{F}) \cdot \Psi(\mathbf{a}) d \mathbf{a}
$$

where the fact that $\mathbf{F}^{T}=-\mathbf{F}$ has again being taken into account.

\section{Suspensions involving deformable clusters}

In this section we are considering a more realistic scenario, that consisting in deformable clusters. The same reasoning as for the rigid cluster will be develop to finally derive the virial stress due to these deformable clusters.

\section{Microscopic kinematics}

Because the cluster is deformable, there's now two kinds of applied forces on each rods bead. The first one is again the one due to the fluid-rod friction once more modelled from:

$$
\mathbf{F}_{i}^{H}=\xi \cdot\left(\nabla \mathbf{v} \cdot \mathbf{p}_{i} \cdot L-\dot{\mathbf{p}}_{i} \cdot L\right)
$$


where the superscript " $H$ " refers to its hydrodynamic nature.

Concerning the second one, we assume a force acting on each bead due to the rods entanglements. This force $\mathbf{F}_{i}^{C}$ is assumed scaling with the difference between the rigid motion velocity (the one that the bead would have if the cluster would be rigid) and the real one:

$$
\mathbf{F}_{i}^{C}=\mu \cdot\left(\mathbf{W} \cdot \mathbf{p}_{i} \cdot L-\dot{\mathbf{p}}_{i} \cdot L\right)
$$

By adding both forces it results:

$$
\mathbf{F}_{i}=\mathbf{F}_{i}^{H}+\mathbf{F}_{i}^{C}=L \cdot\left((\mu \cdot \mathbf{W}+\xi \cdot \nabla \mathbf{v}) \cdot \mathbf{p}_{i}-(\xi+\mu) \cdot \dot{\mathbf{p}}_{i}\right)
$$

that can be rewritten as:

$$
\mathbf{F}_{i}=L \cdot(\xi+\mu) \cdot\left(\frac{\mu \cdot \mathbf{W}+\xi \cdot \nabla \mathbf{v}}{\xi+\mu} \cdot \mathbf{p}_{i}-\dot{\mathbf{p}}_{i}\right)
$$

In particular the rod rotary velocity results:

$$
\begin{aligned}
\dot{\mathbf{p}}_{i}= & \frac{\mu}{\xi+\mu} \cdot \mathbf{W} \cdot \mathbf{p}_{i}+\frac{\xi}{\xi+\mu} \cdot \nabla \mathbf{v} \cdot \mathbf{p}_{i}-\frac{\xi}{\xi+\mu}\left(\mathbf{p}_{i}^{T} \cdot \mathbf{D} \cdot \mathbf{p}_{i}\right) \cdot \mathbf{p}_{i}= \\
& =\frac{\xi}{\xi+\mu} \cdot \dot{\mathbf{p}}_{i}^{J}+\frac{\mu}{\xi+\mu} \cdot \mathbf{W} \cdot \mathbf{p}_{i}=\frac{\xi}{\xi+\mu} \cdot \dot{\mathbf{p}}_{i}^{J}+\frac{\mu}{\xi+\mu} \cdot \dot{\mathbf{p}}_{i}^{R}
\end{aligned}
$$

where $\dot{\mathbf{p}}_{i}^{J}$ is the Jeffery's dilute contribution and $\dot{\mathbf{p}}_{i}^{R}$ the one coming from the rigid cluster contribution.

We can notice that when $\xi \gg \mu$ the cluster does not resist the flow induced rod orientation, and in that case $\dot{\mathbf{p}}_{i} \approx \dot{\mathbf{p}}_{i}^{J}$. In the opposite case, $\mu \gg \xi$, the cluster is too rigid and the bead adopts the velocity dictated by the rigid cluster kinematics $\dot{\mathbf{p}}_{i} \approx \dot{\mathbf{p}}_{i}^{R}$.

\section{Mesoscopic description}

We assume now, as for the rigid cluster, that the deformable cluster is described by the orientation distribution $\psi(\mathbf{p})$ of the rods. As previously, in order to describe the evolution of the suspension, we will consider the evolution of the second order moment of this distribution.

We start considering again the usual second order moment:

$$
\mathbf{a}=\int_{\mathcal{S}(0,1)} \mathbf{p} \otimes \mathbf{p} \cdot \psi(\mathbf{p}) d \mathbf{p}
$$

whose time derivative reads

$$
\dot{\mathbf{a}}=\int_{\mathcal{S}(0,1)}(\dot{\mathbf{p}} \otimes \mathbf{p}+\mathbf{p} \otimes \dot{\mathbf{p}}) \cdot \psi(\mathbf{p}) d \mathbf{p}
$$

where as just deduced the microscopic velocity is given by:

$$
\dot{\mathbf{p}}=\frac{\xi}{\xi+\mu} \cdot \dot{\mathbf{p}}^{J}+\frac{\mu}{\xi+\mu} \cdot \dot{\mathbf{p}}^{R}
$$

that introduced into the expression of the second order moment time derivative results

$$
\dot{\mathbf{a}}=\frac{\xi}{\xi+\mu} \cdot \dot{\mathbf{a}}^{J}+\frac{\mu}{\xi+\mu} \cdot \dot{\mathbf{a}}^{R}
$$

with $\dot{\mathbf{a}}^{J}$ and $\dot{\mathbf{a}}^{R}$ given by: 


$$
\left\{\begin{array}{l}
\dot{\mathbf{a}}^{J}=\Omega \cdot \mathbf{a}-\mathbf{a} \cdot \Omega+\mathbf{D} \cdot \mathbf{a}+\mathbf{a} \cdot \mathbf{D}-2 \cdot \mathbf{A}: \mathbf{D} \\
\dot{\mathbf{a}}^{R}=\mathbf{W} \cdot \mathbf{a}+\mathbf{a} \cdot \mathbf{W}^{T}=\Omega \cdot \mathbf{a}-\mathbf{a} \cdot \Omega+\mathbf{F} \cdot \mathbf{a}-\mathbf{a} \cdot \mathbf{F}
\end{array}\right.
$$

where $\mathbf{A}$ is the fourth order moment of the distribution.

Introducing Eq. (30) into Eq. (29) and grouping the different terms it results:

$$
\dot{\mathbf{a}}=\boldsymbol{\Omega} \cdot \mathbf{a}-\mathbf{a} \cdot \boldsymbol{\Omega}+\frac{\xi}{\xi+\mu} \cdot(\mathbf{D} \cdot \mathbf{a}+\mathbf{a} \cdot \mathbf{D}-2 \cdot \mathbf{A}: \mathbf{D})+\frac{\mu}{\xi+\mu} \cdot(\mathbf{F} \cdot \mathbf{a}-\mathbf{a} \cdot \mathbf{F})
$$

We prove that the model just derived, incorporating drag and network effects, is fully objective.

\section{Global description of a suspension involving several deformable clusters}

As for the case of the rigid clusters, we assume that a microstructure involving suspensions of several deformable clusters can be based on a distribution function $\Psi(\mathbf{x}, t, \mathbf{a})$ giving the fraction of clusters that at position $\mathbf{x}$ and time $t$ have a configuration described by a related to the orientation of the rods composing it and that was noted by $\psi(\mathbf{p})$.

\section{Virial stress induced by deformable clusters suspension}

The virial stress associated with a deformable cluster is computed by considering the hydrodynamic forces acting on each bead $L \cdot \mathbf{p}$

$$
\mathbf{F}^{H}(\mathbf{p})=\xi \cdot L \cdot(\nabla \mathbf{v} \cdot \mathbf{p}-\dot{\mathbf{p}})
$$

from

$$
\boldsymbol{\tau}_{i}=L \cdot \int_{\mathcal{S}(0,1)} \mathbf{p} \otimes \mathbf{F}^{H}(\mathbf{p}) \cdot \psi_{i}(\mathbf{p}) d \mathbf{p}
$$

where the subindex " $i$ " refer to the contribution of cluster $i$ located around point $\mathbf{x}$ and time $t$ where the stress is being computed.

If we introduce now into the expression of the hydrodynamic force the expression of the rod velocity given by Eq. (25) the expression of the hydrodynamic forces reduces to:

$$
\mathbf{F}^{H}(\mathbf{p})=\xi \cdot L \cdot\left(\frac{\mu}{\xi+\mu} \cdot(\mathbf{D} \cdot \mathbf{p}-\mathbf{F} \cdot \mathbf{p})+\frac{\xi}{\xi+\mu}\left(\mathbf{p}^{T} \cdot \mathbf{D} \cdot \mathbf{p}\right) \cdot \mathbf{p}\right)
$$

from with the cluster virial becomes:

$$
\begin{aligned}
& \boldsymbol{\tau}_{i}=L \cdot \int_{\mathcal{S}(0,1)} \mathbf{p} \otimes \mathbf{F}^{H}(\mathbf{p}) \cdot \psi_{i}(\mathbf{p}) d \mathbf{p}= \\
& =2 \cdot N_{p} \cdot\left(\frac{\mu}{\xi+\mu} \cdot \boldsymbol{\tau}_{i}^{R}+\frac{\xi}{\xi+\mu} \cdot \boldsymbol{\tau}_{i}^{J}\right)
\end{aligned}
$$

where the cluster and hydrodynamic contributions, $\tau_{i}^{R}$ and $\tau_{i}^{J}$, read:

$$
\boldsymbol{\tau}_{i}^{R}=\mathbf{D} \cdot \mathbf{a}-\mathbf{F} \cdot \mathbf{a}
$$

and

$$
\boldsymbol{\tau}_{i}^{J}=\mathbf{A}: \mathbf{D}
$$

respectively. 


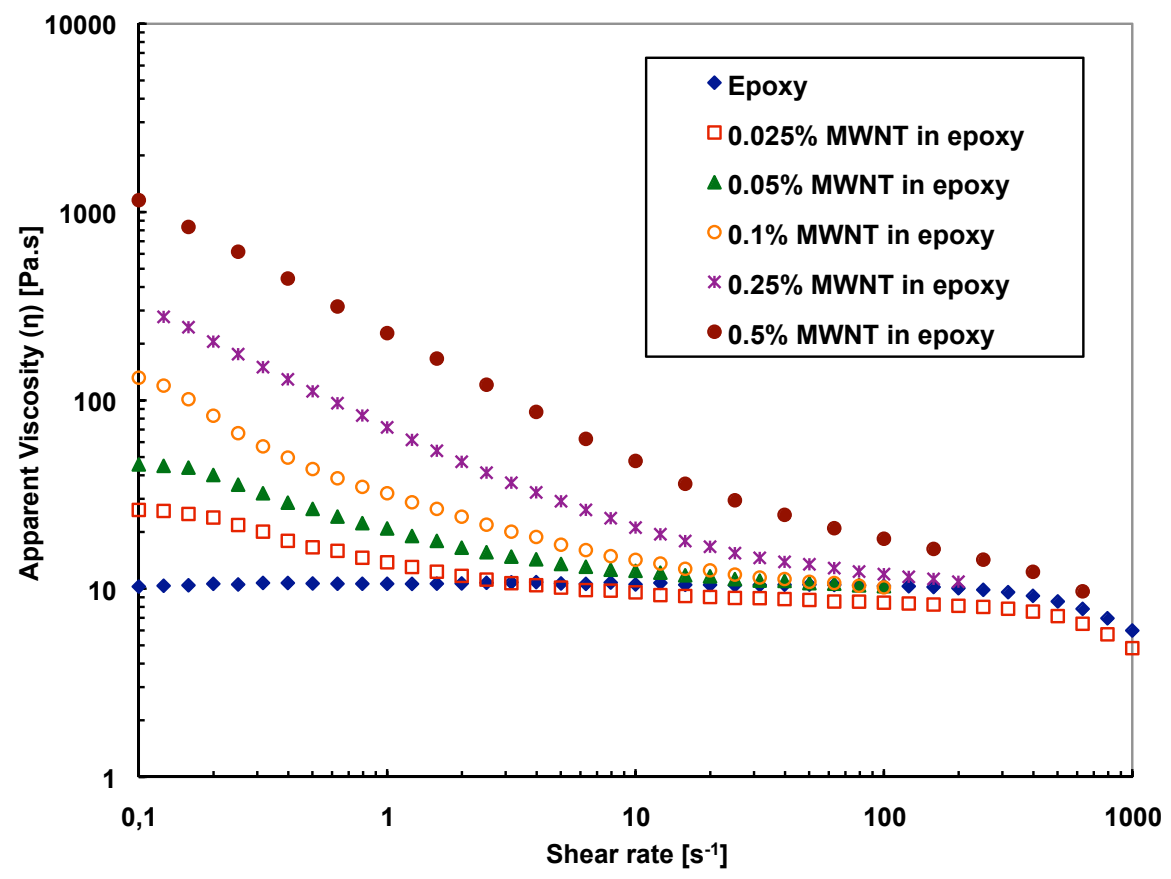

Fig. 6: Evolution of the apparent viscosity of the charged fluid with the shear rate $\dot{\gamma}$

Coming back to the two limits cases previously addressed: the Jeffery's regime is reached by assuming the cluster contribution negligible, in the opposite way, when cluster effects is dominant, the virial stress related to a rigid cluster is obtained.

\section{Study of the behaviour of a charged epoxy}

In order to test the results given by the model presented in the previous part, some numerical results are compared to experimental results reported in Cambridge [6].

This experimental campaign focused on the evolution of the apparent viscosity of a suspension of CNTs into an epoxy matrix in a simple shear flow with the velocity field defined by $(u, v)=(\dot{\gamma} y, 0)$. Results show that the apparent viscosity of the fluid decrease with the increase of the shear rate $\dot{\gamma}$ (Fig. 6).

The idea is there to compare this results to the results issued from the model of deformable clusters.

To do so, in our model, we define the apparent viscosity $\eta_{a p}$ as:

$$
\eta_{a p}=\frac{\boldsymbol{\tau}_{i}}{\dot{\gamma}}
$$

Now, considering this apparent viscosity, the equations derived in the previous part show that the relation between $\boldsymbol{\tau}_{i}$ and $\dot{\gamma}$ (related to $\nabla \mathbf{v}$ ) is linear. Then, our model can not describe the experimental results presented in Fig. 6. We then have to introduce a new ingredient in the model to mimic the results. To do so, let's come back to the physics of the behaviour of such suspensions.

As seen before, the deformation of a cluster results from the competition between two forces: the one due to the external fluid flow that tends to align the rods composing the cluster in the flow direction (the hydrodynamic force), and the "internal" one, linked to the resistance of the cluster to deform.

In the experiments, increasing the value of $\dot{\gamma}$ comes to the same thing as reinforcing the action of the hydrodynamic forces in the flow direction, and then tend to facilitate the alignment of the rods in 
the cluster. And, a cluster with all rods are almost in the same direction does not create much internal stress because it does not resist the flow.

We can then say that the relevant phenomenon here to explain such experimental results is the competition between the two forces applied on clusters.

In the model developed previously, the competition between these two forces is described by the ratio between the two coefficients $\xi$ and $\mu$.

In order to introduce the non linear dependency of the apparent viscosity with the shear rate $\dot{\gamma}$, we derive a law that define the evolution of the ratio between $\xi$ and $\mu$ to mimic the experimental results. The friction coefficient $\xi$ depends on the nature of the solvent and the suspended particles, so it must be considered fixed. However, the parameters describing the cluster rigidity $\mu$ can depend on the applied shear, and we can imagine that it could decrease when the rods aligning in the flow direction because the decrease of the entanglements density.

Remark: calculation of the apparent viscosity Depending on the initial orientation distribution used for the calculation and the values of $\xi, \mu$ and $\dot{\gamma}$, the apparent viscosity varies in time. Generally, when $\mu>>\xi$, the apparent viscosity is periodic due to the fact that we approach a rigid cluster behaviour with an orientation evolving periodically in time. On the opposite, when $\mu<<\xi$, the apparent viscosity varies until reaching the constant null value, once all the clusters are aligned in the flow direction. Between these two behaviours, one can prove that a steady state exists, leading to a constant apparent viscosity. This particular behaviour is obtained for the following initial conditions:

$$
\mathbf{a}=\left(\begin{array}{cc}
\frac{\mu-\xi}{2 \mu} & 0 \\
0 & \frac{\mu+\xi}{2 \mu}
\end{array}\right)
$$

Identification of the evolution of $\mu$ with $\dot{\gamma}$ In Fig. 7, one can see the values of $\mu$ identified for the different values of shear rate. From this result, we can derive a power function representing the evolution of the parameter $\mu$ with the shear rate:

$$
\mu(\dot{\gamma})=9 \cdot 10^{-4} \dot{\gamma}^{-3,8}+0,55
$$

The comparison between the experimental results and the numerical prediction of our model have shown that because the model does integrate the main physical phenomenon that can be observed for such suspensions, it can mimic experimental observations as soon as the identification of the parameters is made carefully.

\section{Conclusions}

In this work, a complete multiscale and physically based model of suspensions involving rigid/deformable clusters is developed. This model introduces parameters, as $\mu$ or $\xi$, allowing to describe the different clusters behaviours observed experimentally. In the last section, the application of this model and its confrontation to experimental results have shown that the identification of these parameters and their dependence on the flow conditions must be explained physically, probably by considering richer physics as cluster coalescence or disagregation.

\section{References}

[1] G.B. Jeffery: Proc. R. Soc. London. Vol A102 (1922) p. 161-179 


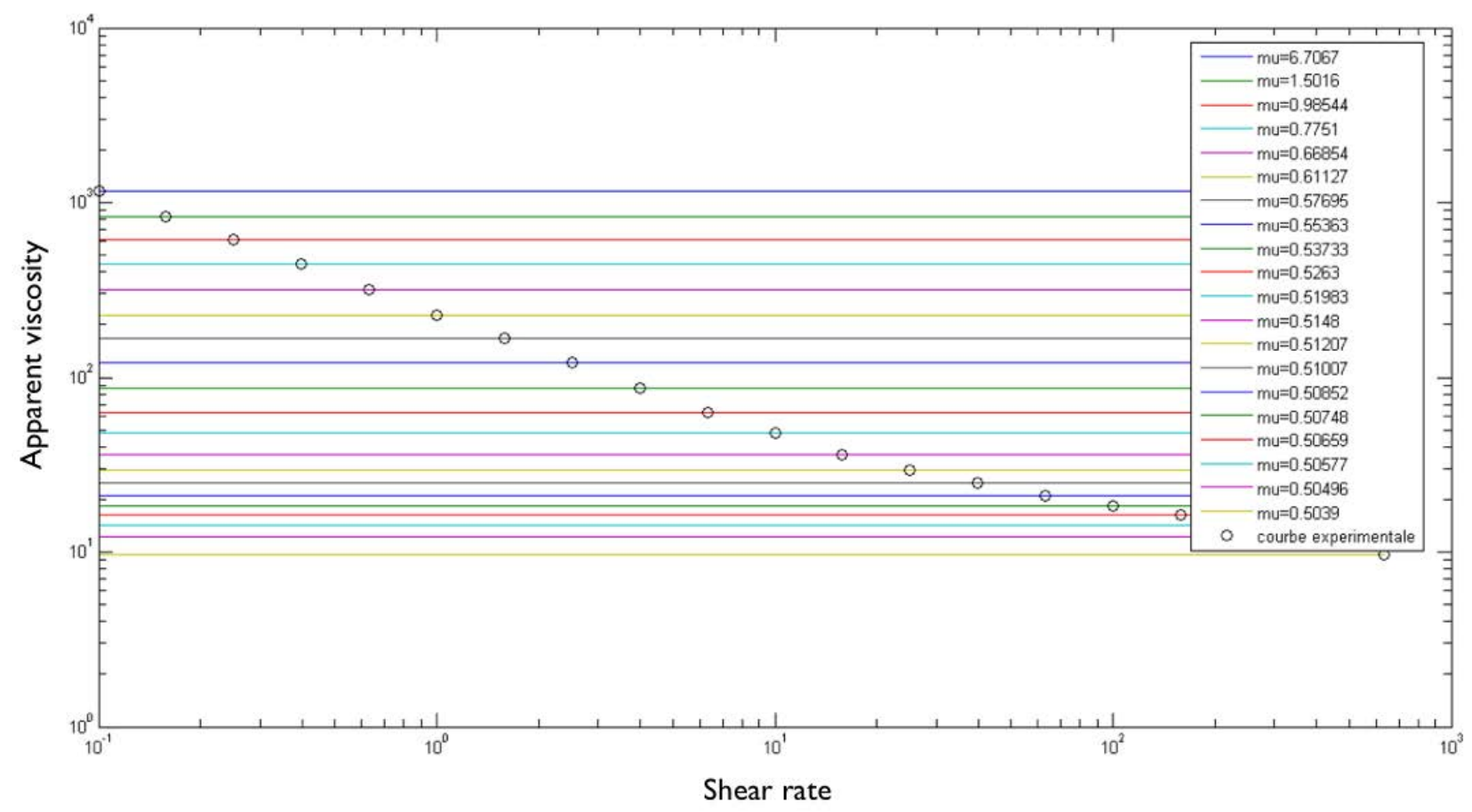

Fig. 7: Identification of the parameter $\mu$ to describe the apparent viscosity dependency with the shear rate (study done for $0,5 \% \mathrm{MWNT}$ in epoxy)

[2] M. Doi, S.F. Edwards: The Theory of Polymer Dynamics (Clarendon Press, Oxford, 1987)

[3] R.B. Bird. C.F. Crutiss, R.C. Armstrong, O. Hassager, in: Dynamic of polymeric liquid, Volume 2: Kinetic Theory edited by John Wiley and Sons (1987)

[4] S. Advani, Ch. Tucker: J. Rheol. Vol. 31 (1987) p. 751-784

[5] F. Chinesta: From Single-Scale to Two-Scales Kinetic Theory Descriptions of Rods Suspensions (Archives in Computational Methods in Engineering, 2012)

[6] A. Ma, F. Chinesta, A. Ammar, M. Mackley: Journal of Rheology Vol. 52 (2008) p. 1311-1330 